\title{
Novel metallic behavior in two dimensions
}

\author{
X. G. Feng ${ }^{1}$, Dragana Popović ${ }^{1}$, S. Washburn ${ }^{2}$, and V. Dobrosavljević ${ }^{1}$ \\ ${ }^{1}$ National High Magnetic Field Laboratory, Florida State University, Tallahassee, FL 32310 \\ ${ }^{2}$ Dept. of Physics and Astronomy, The University of North Carolina at Chapel Hill, Chapel Hill, NC 27599
}

(September 1, 2018)

\begin{abstract}
Experiments on a sufficiently disordered two-dimensional (2D) electron system in silicon reveal a new and unexpected kind of metallic behavior, where the conductivity decreases as $\sigma\left(n_{s}, T\right)=\sigma\left(n_{s}, T=0\right)+A\left(n_{s}\right) T^{2}\left(n_{s}\right.$ - carrier density) to a non-zero value as temperature $T \rightarrow 0$. In $2 \mathrm{D}$, the existence of a metal with $d \sigma / d T>0$ is very surprising. In addition, a novel type of a metal-insulator transition obtains, which is unlike any known quantum phase transition in $2 \mathrm{D}$.
\end{abstract}

PACS Nos. 71.30.+h, 71.27.+a, 73.40.Qv

The transport behavior indicative of the existence of a metallic state at zero temperature in two dimensions (2D) has been observed in a variety of 2D electron [1] and hole [1] systems. However, there is still no generally accepted microscopic description of the apparent metallic phase and the metal-insulator transition (MIT) in 2D. Until now, the metallic phase has been characterized by an increase of conductivity $\sigma$ as temperature $T \rightarrow 0$. In the presence of a particular type of disorder, such a metallic phase is suppressed [5]. Here we show that this gives rise to a new and unexpected kind of metallic behavior, where $\sigma$ decreases but does not go to zero (as expected for an insulator) when $T \rightarrow 0$. While unambiguously established by our data, this behavior is in a striking contradiction with any theoretical description available to date. In addition, we report the discovery of a novel type of a MIT, which occurs between this metal and an insulating state as the carrier density $n_{s}$ is reduced. While the transition is analogous to that observed in some three-dimensional (3D) systems, it is unlike any known quantum phase transition in $2 \mathrm{D}$. We point out that our samples are representative of a broad class of $\mathrm{Si}$ metal-oxide-semiconductor field-effect transistors (MOSFETs) historically known as "nonideal" samples 6].

In Si MOSFETs, the 2D electron system is formed by confining the electrons to the $\mathrm{Si}$ side of the $\mathrm{Si}_{-} \mathrm{SiO}_{2}$ interface. At low $T$, electrons are quantized in the lowest energy level (subband) for motion perpendicular to the interface, and are thus forced to move in a plane parallel to it. $n_{s}$ is varied by applying voltage $V_{g}$ to the metallic gate. As a result of disorder (potential scattering by the charges in the oxide and by the roughness of the interface), the 2D density of states of each subband acquires a tail of strongly localized states. In sufficiently disordered samples, such as ours, the band tails may be so long that some of the localized states in the tail of the upper subband [7] may be populated even at low $n_{s}$, and act as additional scattering centers for $2 \mathrm{D}$ electrons [8]. In particular, since at least some of them must be singly populated due to a large on-site Coulomb repulsion (tens of $\mathrm{meV}$ ), it is plausible that they may act as local magnetic moments. We have shown earlier [5] that an arbitrarily small amount of such scattering suppresses the $d \sigma / d T<0$ behavior in the $T \rightarrow 0$ limit. We note that, strictly speaking, since the carriers in the ground subband can exchange with the carriers in the tail of the upper subband, there is some hybridization between the two but the important question is the time scale for the various different mechanisms $(e . g$. for the exchange and for the mean free time for transport). We also point out that some evidence for qualitatively the same behavior of $\sigma(T)$ was found [5] even in the case when only the ground subband was occupied but the potential scattering was increased considerably by applying large negative voltage $\left(V_{s u b}\right)$ to the Si substrate. The results were interpreted as being due to the formation of disorder-induced local moments (singly occupied localized states) associated with the ground subband. We realize, however, that the detailed microscopic picture is probably more complex, as discussed below.

By varying $V_{\text {sub }}$, the splitting of the subbands and hence the population of the tail of the upper subband are changed. In the presence of occupied "upper tail" states, in the metallic phase $d \sigma / d T$ changes sign from positive to negative at $T=T_{m}\left(n_{s}, V_{\text {sub }}\right)$ as $T$ increases [5]. For $T>T_{m}$, we observe [2,5] the "conventional" $2 \mathrm{D}$ metallic behavior with $d \sigma / d T<0$ and other features (e. g. scaling) that reflect the "conventional" [1] MIT. The focus of this study, however, is the low- $T\left(T<T_{m}\right)$ regime. We concentrate on the data obtained with $V_{s u b}=+1 \mathrm{~V}$, because the reduced subband splitting leads to a large number of occupied "upper tail" states and $T_{m}>4.5 \mathrm{~K}$, and hence to the observation of the novel behavior of $\sigma$ up to relatively high $T(\sim 2 \mathrm{~K})$. The same qualitative behavior is also observed when the number of occupied "upper tail" states is smaller (e.g. for negative $\left.V_{\text {sub }}\right)$, the only difference being that the low- $T\left(T<T_{m}\right)$ regime becomes smaller 5 . For $V_{\text {sub }}=+1 \mathrm{~V}$, the number of populated states in the tail of the upper subband is roughly constant for all $n_{s}$ that are of interest here $\left(n_{s} \lesssim\right.$ $n_{\max } \sim 5 \times 10^{15} \mathrm{~m}^{-2}$, where $n_{\max }$ is the density where mobility reaches its maximum) [9]. 

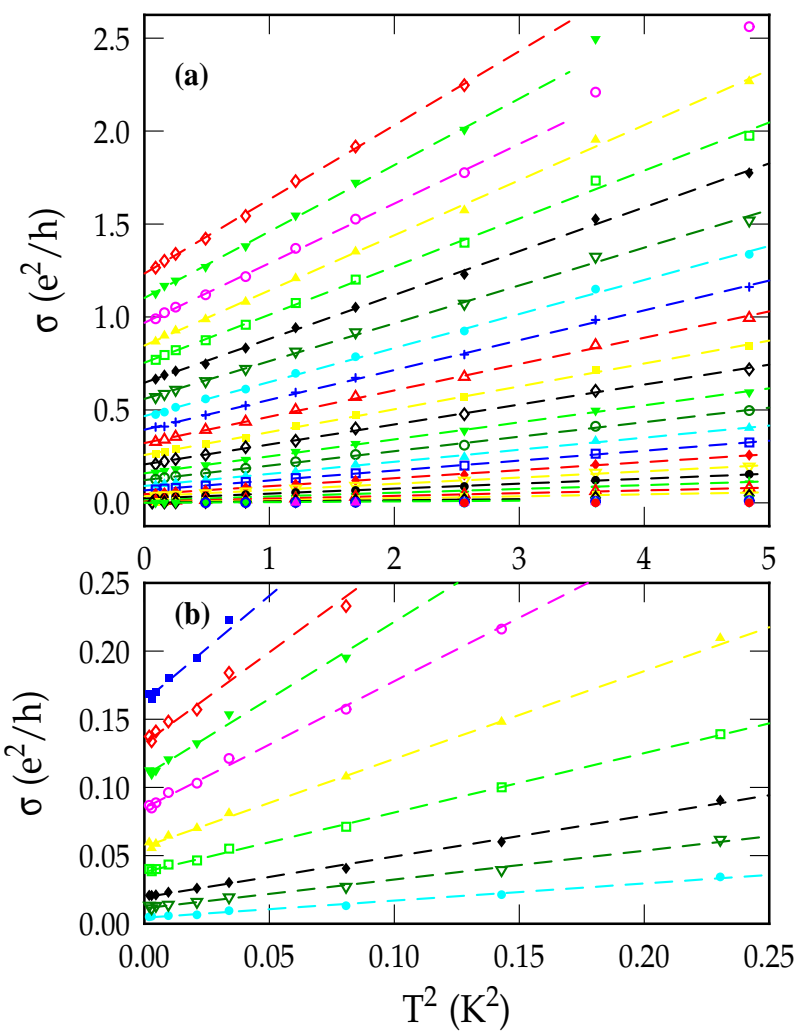

FIG. 1. $\sigma(T)$ plotted vs. $T^{2}$ for different $n_{s}$. The dashed lines are fits. (a) Sample 12: $n_{s}$ varies from $3.0 \times 10^{15} \mathrm{~m}^{-2}$ (top) down to $0.7 \times 10^{15} \mathrm{~m}^{-2}$ (bottom) in steps of $0.1 \times 10^{15} \mathrm{~m}^{-2}$ and $0.3 \leq T<2.2 \mathrm{~K}$. (b) Sample 11: top to bottom, $n_{s}=3.8,3.7,3.6 \times 10^{15} \mathrm{~m}^{-2}$, and $3.5 \times 10^{15} \mathrm{~m}^{-2}$ to $2.5 \times 10^{15} \mathrm{~m}^{-2}$ in steps of $0.2 \times 10^{15} \mathrm{~m}^{-2} ; 0.045 \leq T<0.5 \mathrm{~K}$.

Our samples are standard two-terminal Si MOSFETs of Corbino (circular) geometry (channel length $=0.4 \mathrm{~mm}$, mean circumference $=8 \mathrm{~mm}$ ), and a peak mobility $\sim 1 \mathrm{~m}^{2} / \mathrm{Vs}$ at $4.2 \mathrm{~K}$. Other sample details have been given elsewhere [2, 10]. Conductance was measured as a function of $V_{g}$ using a low-noise current preamplifier and a low-noise analog lock-in at $\sim 13 \mathrm{~Hz}$ (the lead resistance was subtracted by the usual method). The excitation voltage $V_{\text {exc }}$ was kept constant and low enough to avoid electron heating [11]. Most of the measurements were carried out in a $H e^{3}$ cryostat (base $T=0.247 \mathrm{~K}$ ). Figure 1 1 (a) shows $\sigma(T)$ for different values of $n_{s}$. An excellent fit (dashed lines) to the data is obtained with

$$
\sigma\left(n_{s}, T\right)=\sigma\left(n_{s}, T=0\right)+A\left(n_{s}\right) T^{2}
$$

over a wide range of $n_{s}$ as shown, where $\sigma$ measured at the lowest $T(0.3 \mathrm{~K})$ varies over three orders of magnitude below $e^{2} / h\left(e^{2} / h\right.$ - quantum unit of conductance). For each $n_{s}$, the best exponent in the power law dependence of $\sigma$ on $T$ [Eq. (11)] lies within $10 \%$ of 2 . Several samples have been studied and they all exhibit qualitatively the same behavior. Fig. 11(b) shows the data obtained on another sample (\# 11) at $T$ down to $0.045 \mathrm{~K}$. Obviously, Eq. (11) describes the data well even at this, an order of

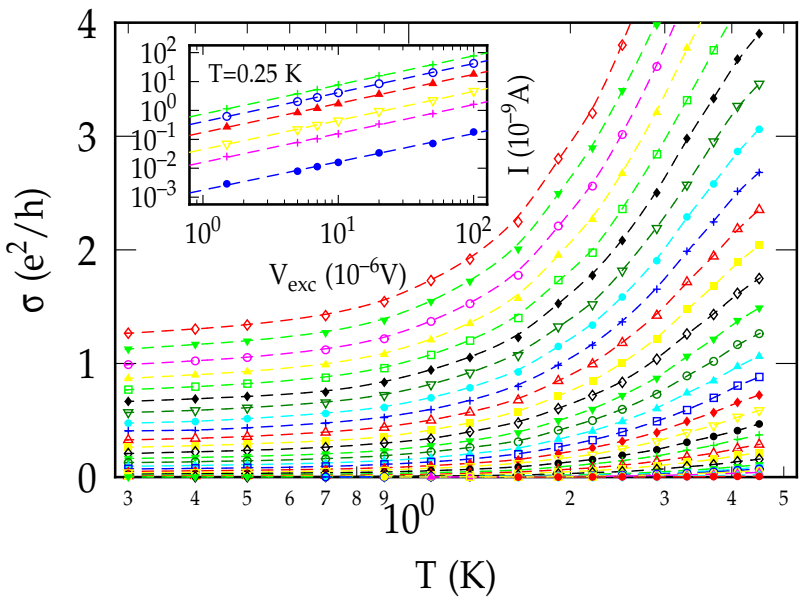

FIG. 2. The same data as in Fig. 1(a) plotted vs. $\ln T$. The dashed lines guide the eye. Inset: Current $I$ (for sample 17) vs. $V_{\text {exc }}$ for $n_{s}\left(10^{15} \mathrm{~m}^{-2}\right)=3.0,2.5,2.0,1.5,1.25,1.0$ (top to bottom). The dashed lines are fits with the slopes equal to 1 .

magnitude lower $T$ [12]. At such low $T$, it is not possible to study the behavior very close to the MIT because of the small signal-to-noise ratio. The observed $\sigma(T)$ spans, therefore, two decades in $T$, starting from $T \sim 2 \mathrm{~K}$, and clearly cannot be explained by heating effects.

In order to compare our data to the predictions of the theory of weak localization [13], we plot the data from Fig. 1(a) vs. $\ln T$. Fig. 2 shows that $\sigma$ cannot be described by $\ln T$ in any range of $T$ and, in fact, the curvature of the observed $\sigma(T)$ is the opposite from the one expected for an insulating state. We note that the conduction at $0.25 \mathrm{~K}$ was ohmic 14 for $V_{\text {exc }}$ of up to at least $100 \mu \mathrm{V}$ (Fig. 2 2 inset) but $V_{\text {exc }} \sim 10 \mu \mathrm{V}$ was typically used at $T \geq 0.25 \mathrm{~K}$.

The high quality of the fits [Eq. (1)] allows a reliable extrapolation of $\sigma\left(n_{s}, T=0\right)$, whose finite (i. e. non-zero) values mean that, in spite of the decrease of $\sigma\left(n_{s}, T\right)$ with decreasing $T$, the $2 D$ system is in the metallic state. $\sigma\left(n_{s}, T=0\right)$ is shown in Fig. 3 for two different samples as a function of $\delta_{n}=\left(n_{s}-n_{c}\right) / n_{c}$, the distance from the MIT ( $n_{c}$ - critical density). For sample 12, for example, the Fermi energy $E_{F} \approx 4 \mathrm{~K}$ at the MIT, and $r_{s} \approx 22$ $\left(r_{s}\right.$ is the average inter-electron separation in units of the effective Bohr radius). We find the power-law behavior $\sigma\left(n_{s}, T=0\right) \sim \delta_{n}^{\mu}(\mu=2.99 \pm 0.01$ for sample $12)$, as expected in the vicinity of a quantum critical point [15], such as the MIT. The power law holds over a very wide range of $\delta_{n}$ (up to 5) similar to what has been observed [16] in Si:P near the MIT. In addition, even though the MIT occurs at different $n_{c}$ in different samples, the critical exponents $\mu$ are the same in both samples (Fig. 3), as expected from general arguments 15].

In addition, very general considerations have suggested 17] that the conductivity near the MIT can be described by a scaling form

$$
\sigma\left(n_{s}, T\right)=\sigma_{c}(T) f\left(T / \delta_{n}^{z \nu}\right),
$$




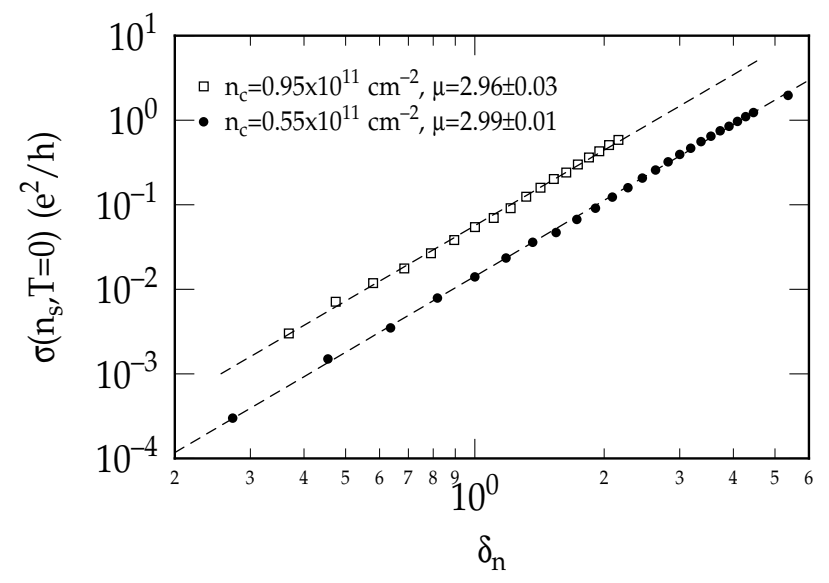

FIG. 3. $\sigma\left(n_{s}, T=0\right)$ vs. the distance from the MIT for samples 9 (squares) and 12 (dots). The dashed lines are fits with the slopes equal to the critical exponent $\mu$.

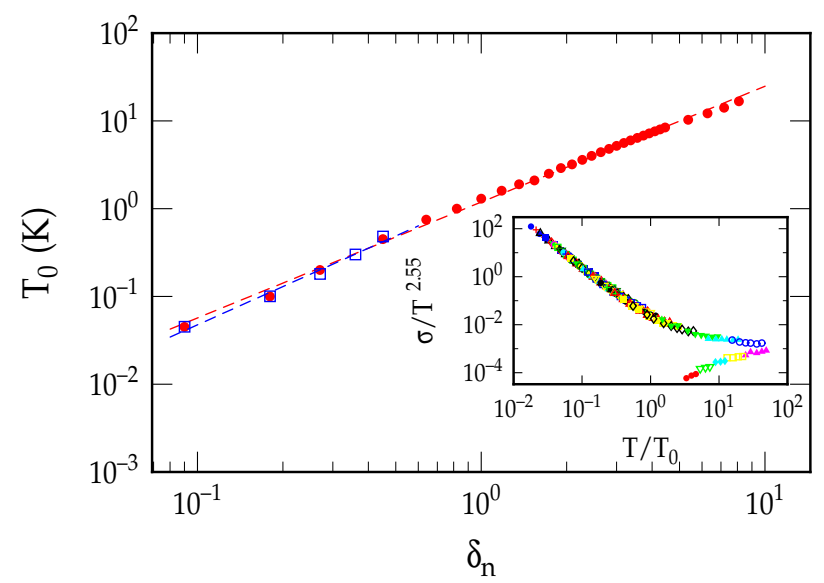

FIG. 4. Scaling parameter $T_{0}$ as a function of $\left|\delta_{n}\right|$ for sample 12. Open symbols correspond to the insulating side of the transition, closed symbols to the metallic one. The dashed lines are fits with slopes $1.4 \pm 0.1$ and $1.32 \pm 0.01$, respectively. Inset, scaling of raw data $\sigma / \sigma_{c} \sim \sigma / T^{x}$ in units of $e^{2} / h \mathrm{~K}^{2.55}$. Different symbols correspond to different $n_{s}$ ranging from $0.3 \times 10^{15} \mathrm{~m}^{-2}$ to $3.0 \times 10^{15} \mathrm{~m}^{-2}$. It was possible to scale all the data below about $2 \mathrm{~K}$.

where $z$ and $\nu$ are the dynamical and correlation length exponents, respectively, and the critical conductivity

$$
\sigma_{c}=\sigma\left(n_{s}=n_{c}, T\right) \sim T^{x} .
$$

Indeed, we find such a power-law dependence, with $x=$ $2.55 \pm 0.3$ for sample 12 , and $x=2.7 \pm 0.5$ for sample 9 .

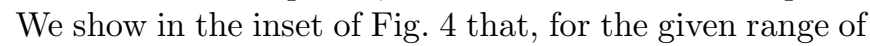
$n_{s}$ and $T$, all $\sigma\left(n_{s}, T\right) / \sigma_{c}(T) \sim \sigma\left(n_{s}, T\right) / T^{x}$ collapse onto a single scaling function $f\left(T / T_{0}\right)$. $T_{0}$ is displayed in Fig. 1 as a function of $\delta_{n}$ on both sides of the transition. Again, we find a power-law behavior, $T_{0} \sim\left|\delta_{n}\right|^{z \nu}$, in agreement with Eq. (2). For the metallic side of the transition, $z \nu=$ $1.32 \pm 0.01$, and for the insulating side $z \nu=1.4 \pm 0.1$. This value of $z \nu$ is similar to $z \nu=0.8-1.7$ obtained [1,2,5, 18 in Si MOSFETs for the 2D MIT that occurs between an insulator and a metal with $d \sigma / d T<0$. We also note that
Eq. (11) describes the data well only in the low- $T$ metallic regime, $i$. e. for $T<T_{0}\left(\delta_{n}\right)$. In general, a different $\sigma(T)$ is expected in the quantum critical regime $\left[T>T_{0}\left(\delta_{n}\right)\right]$, as given by Eq. (3) [17], and this is exactly what we find. For example, for $0.3 \leq T<2 \mathrm{~K}$, all data for $\delta_{n} \lesssim 0.25$ in Fig. 11(a) follow Eq. (3) (i.e. $\left.\sigma\left(n_{s}, T\right) \sim T^{x}\right)$.

It is easy to show using standard scaling arguments 17] that $\mu=x(z \nu), i$. $e$. that $\mu$ can be determined not only from extrapolations of $\sigma(T)$ to $T=0$ (see Eq. (1) and Fig. (3) but also based on all data taken at all $T$ and values of $n_{s}$ for which scaling holds. In this way, we obtain $\mu=x(z \nu)=3.4 \pm 0.4$ (for sample 12), in agreement with the method used above. We note that the uncertainty in $\mu$ obtained from dynamical scaling is comparable in size to those of various critical exponents in other systems $(\approx 5-30 \%)[19$. By contrast, $\mu$ found from zerotemperature extrapolation of $\sigma(T)$ is determined with a much higher accuracy, to within $0.3 \%$ (Fig. 3). This is especially remarkable since $\sigma\left(n_{s}, T=0\right)$ (for sample 12) spans four orders of magnitude.

Several striking features of our data stand out.

(1) $\sigma(T)$ follows a precise $T^{2}$ form over a very broad $T$ range. Such behavior is well established for metals containing local magnetic moments, and is believed to result from the Kondo effect 20]. In fact, to the best of our knowledge, there is no other known mechanism that results in an increase of $\sigma$ as $T^{2}$. In our case, this feature provides the most direct evidence of the presence of local magnetic moments. It is worth noticing, however, that exchange with any kind of two-level systems is capable of mimicking much of the Kondo effect [21]. Even though there may be some such shallow traps present at the $\mathrm{Si}-\mathrm{SiO}_{2}$ interface, it is difficult to rationalize the nonmonotonic dependence on $V_{\text {sub }}$ that we observe [5] based on any interface trap model. If the two-level systems are indeed responsible for the observed $\sigma(T)$, it is more likely that they are related to the occupied states in the tail of the upper subband [22]. In general, one expects the $T^{2}$ behavior for a quantum impurity embedded in a Fermi liquid in any dimension. On the other hand, it has been suggested [23] that the metallic state in 2D may not have a Fermi liquid character, but this interesting question requires further theoretical and experimental work. In particular, careful experiments in a parallel magnetic field should provide valuable insights into the microscopic nature of the conduction mechanism.

We point out that the very existence of the MIT and the associated dependence of various quantities on $n_{s}$ near the critical region, reflect the importance of the localization effects in addition to the interaction mechanisms such as the Kondo effect. From a practical point of view and regardless of the interpretation, such a simple $\sigma(T)$ [Eq. (1D)] is crucial for characterizing the low- $T$ state of the system since it allows for an unambiguous extrapolation to $T=0$.

(2) Scattering of the 2D conduction electrons by elec- 
trons populating the tail of the upper subband leads to a qualitative modification of the low- $T$ transport, and to a novel metallic behavior characterized by $d \sigma / d T>0$. Our data demonstrate clearly that, contrary to what is usually assumed, the mere decrease of $\sigma$ with decreasing $T$ at a given $n_{s}$ does not necessarily imply the existence of an insulating state at $T=0$. Hence, for example, without a detailed study of $\sigma(T)$ and without any evidence for scaling, the observed 24] change from $d \sigma / d T<0$ to $d \sigma / d T>0$ at high $n_{s}$ does not represent an evidence for the second MIT as $n_{s}$ is varied.

(3) In the 2D metal studied here, $\sigma\left(n_{s}, T=0\right) d e$ creases continuously, and follows a distinct power-law behavior as the MIT is approached. In particular, metallic $\sigma$ as small as $10^{-3} e^{2} / h$ has been observed, in a striking contrast to anything that has been reported in other $2 \mathrm{D}$ systems when $d \sigma / d T<0$. A similar observation in $3 \mathrm{D}$ systems 16] has demonstrated the absence of minimum metallic conductivity, and has had a profound impact on shaping the theoretical ideas about the MIT.

(4) Our data show an excellent fit to the dynamical scaling described by Eq. (2). It should be noted that such scaling behavior is not consistent with the simple singleparameter scaling hypothesis [23]. In particular, this formulation 23] allows only for a "metallic-like" temperature dependence $(d \sigma / d T<0)$ in the conducting phase, in contrast to what we find. In addition, the exponent $x$ in Eq. (3) is expected to take the value $x=(D-2) / z$, and thus to vanish in 2D. On the other hand, it should be emphasized that $x \neq 0$ does not contradict [17] any fundamental principle even for 2D systems. Indeed, such violations of "Wegner scaling" [17] were predicted for certain microscopic models in presence of "dangerously irrelevant operators" 25. Interestingly, recent work on the 2D MIT for a $d \sigma / d T<0$ metal has found evidence 26. 22. that the same scaling form can be used to resolve some apparent violations of the single-parameter scaling. In that case though, the exponent $x$ takes a distinctly different value, presumably reflecting the different universality classes of the two situations.

Our study shows that, in the presence of a particular kind of disorder, a new type of metallic behavior and a MIT are exhibited in a $2 \mathrm{D}$ electron system. In contrast to the $2 \mathrm{D}$ conducting phase and the MIT discovered by Kravchenko et al. [1], the 2D metal studied here has $d \sigma / d T>0$, and the scaling form that describes the conductivity near the MIT [Eq. (2)] includes a T-dependent prefactor $\sigma_{c}(T)$, analogous to the MIT in 3D systems.

We are grateful to K. Eng for technical assistance. This work was supported by NSF Grant DMR-9796339, NHMFL through NSF Cooperative Agreement DMR9527035, an NHMFL In-House Research Program grant (D. P.), NSF Grant DMR-9974311 (V. D.), and Alfred P. Sloan Foundation (V. D.).
[1] S. V. Kravchenko, et al., Phys. Rev. B 50, 8039 (1994); S. V. Kravchenko, et al. Phys. Rev. B 51, 7038 (1995).

[2] D. Popović, et al., Phys. Rev. Lett. 79, 1543 (1997).

[3] S. Papadakis and M. Shayegan, Phys. Rev. B 57, R15068 (1998); Y. Hanein, et al., Phys. Rev. B 58, R13338 (1998); E. Ribeiro, et al., Phys. Rev. Lett. 82, 996 (1999).

[4] J. Lam, et al., Phys. Rev. B 56, R12741 (1997); P. T. Coleridge, et al., Phys. Rev. B 56, R12764 (1997); Y. Hanein, et al., Phys. Rev. Lett. 80, 1288 (1998); M. Y. Simmons, et al., Phys. Rev. Lett. 80, 1292 (1998).

[5] X. G. Feng, et al., Phys. Rev. Lett. 83, 368 (1999).

[6] T. Ando, A. B. Fowler, and F. Stern, Rev. Mod. Phys. 54, 437 (1982), and references therein.

[7] Usually, this is a tail of the $E_{0^{\prime}}$ subband - the lowest subband associated with the four conduction band valleys with the light effective mass $\left(0.190 m_{e}\right)$ perpendicular to the interface. See also Ref. [6].

[8] A. B. Fowler, Phys. Rev. Lett. 34, 15 (1975); A. Kastalsky and F. F. Fang, Surf. Sci. 113, 153 (1982); U. Kunze, J. Phys. C 17, 5677 (1984); S. Kawaji and N. Nagashima, Surf. Sci. 196, 316 (1988); D. Popović, F. F. Fang, and P. J. Stiles, Solid State Commun. 68, 25 (1988).

[9] D. Popović, et al., in "Quantum Physics at Mesoscopic Scale", ed. by D. C. Glattli, M. Sanquer, and J. Tran Tranh (Editions Frontieres, Dreux, 1999).

[10] Measurements in the quantum Hall regime indicate that contact resistances are less than a few Ohms, and the response to $V_{\text {sub }}$ [5] proves that the measured effects arise from the $2 \mathrm{D}$ channel - not the contacts.

[11] For example, at the lowest bath $T(0.020 \mathrm{~K})$, where the sample was immersed in the mixing chamber of a $\mathrm{He}^{3}-$ $H e^{4}$ dilution refrigerator, $V_{\text {exc }} \sim 1 \mu \mathrm{V} \mathrm{rms}(\mathrm{eV}=0.011 \mathrm{~K}$ $<k T)$, so the total power dissipated in the sample was only between $10^{-16} \mathrm{~W}$ and $10^{-14} \mathrm{~W}$, depending on $n_{s}$.

[12] Another sample (\#19) obeyed Eq. (1) down to $0.020 \mathrm{~K}$.

[13] See P. A. Lee and T. V. Ramakrishnan, Rev. Mod. Phys. 57, 287 (1985).

[14] Amplitudes for any possible quadratic terms are statistically insignificant.

[15] N. Goldenfeld, Lectures on Phase Transitions and the Renormalization Group (Addison-Wesley, 1992).

[16] T. F. Rosenbaum, et al., Phys. Rev. Lett. 45, 1723 (1980).

[17] D. Belitz, et al., Rev. Mod. Phys. 66, 261 (1994).

[18] S. V. Kravchenko, et al., Phys. Rev. Lett. 77, 4938 (1996); V. M. Pudalov, et al., JETP Lett. 68, 442 (1998).

[19] M. P. Sarachik, in Metal-Insulator Transitions Revisited, edited by Edwards, P. P. \& Rao, C. N. (Taylor and Francis, London, 1995), and references therein.

[20] A. C. Hewson, The Kondo Problem to Heavy Fermions (Cambridge Univ. Press, Cambridge, England, 1993).

[21] K. Vladár, et al., Phys. Rev. B 28, 1596 (1983).

[22] S. Washburn, et al., Ann. Phys. (Leipzig) 8, 569 (1999).

[23] V. Dobrosavljević, et al., Phys. Rev. Lett. 79, 455 (1997).

[24] V. M. Pudalov, et al., JETP Lett. 68, 534 (1998); A. R. Hamilton, et al. Phys. Rev. Lett. 82, 1542 (1999); V. M. Pudalov, et al., Phys. Rev. B 60, R2154 (1999).

[25] C. Castellani, et al., Phys. Rev. Lett. 59, 323 (1987); T. R. Kirkpatrick, et al., Phys. Rev. Lett. 74, 1178 (1994); D. Belitz, et al., Z. Phys. B 98, 513 (1995).

[26] N.-J. Kim, et al., preprint cond-mat/9809357 (1998). 